\title{
The Near-to-Native-State Architecture of Measles Virus Assembly Sites and Isolated Measles Virus Particles
}

Elizabeth R. Wright ${ }^{1}$, Joshua D. Strauss ${ }^{1}$, Zunlong, $\mathrm{Ke}^{2}$, Cheri M. Hampton ${ }^{1}$, Fredrick Leon ${ }^{1}$, Melinda Brindley $^{3}$, and Richard K. Plemper ${ }^{4}$.

${ }^{1 .}$ Department of Pediatrics, Emory University School of Medicine, and Children's Healthcare of Atlanta, Atlanta, Georgia, USA.

2. School of Biology, Georgia Institute of Technology, Atlanta, Georgia, USA.

${ }^{3 .}$ Department of Infectious Diseases, College of Veterinary Medicine, The University of Georgia, Athens, Geogia, USA.

4. Institute for Biomedical Sciences, Georgia State University, Atlanta, Georgia, USA.

Measles virus $(\mathrm{MeV})$ is a pleomorphic, enveloped, single-stranded RNA virus that belongs to the Paramyxovirus family. Details regarding the coordination and native arrangement of the Measles virus macromolecular complexes at sites of virus assembly and in released virus particles are poorly understood. Three-dimensional structural analyses of Measles viruses by cryo-electron tomography (cryo-ET) have been of purified viruses [1,2]. To further investigate the structure of assembled, released Measles virus particles and the processes of virus assembly under native-state conditions, we utilized whole cellular cryo-ET.

HeLa and MRC-5 cells were grown directly on gold Quantfoil TEM grids (Quantifoil Micro Tools $\mathrm{GmbH}$, Jena, Germany). Subsequently, cells were infected with MeV strains, including wild type and recombinant strains at a multiplicity of infection of 1,6 or 10. TEM grids were plunge frozen in liquid ethane using a Cryoplunge 3 system (Gatan, Pleasanton, CA) as previously described [4, 5]. Cryo-grids were imaged with a JEOL JEM-2200FS TEM (JEOL, Ltd., Japan) equipped with an in-column Omega energy filter, operated with a slit with of $20 \mathrm{eV}$. Image montages were acquired at a magnification of $10,000 \times$, equivalent to a pixel size of $6.14 \AA$ on a DE-20 direct electron detector camera (Direct Electron, LP, San Diego, CA). Bidirectional tilt-series were acquired at $2^{\circ}$ increments over an angular range of $-62^{\circ}$ to $+62^{\circ}$ using the SerialEM software package [6]. Images were recorded at a magnification of $20,000 \times$, corresponding to a pixel size of $2.94 \AA$ on a DE-20 direct electron detector camera at a rate of 12 frames per second. Data was processed as previously described [3] and tomograms were reconstructed using the IMOD software package [7].

Thin areas along the periphery of Measles virus-infected cells were surveyed to locate sites of virus assembly and to identify released virus particles. Regions of interest were identified and cryo-ET data was acquired. Sites of assembly were identified by the presence of the matrix protein along the plasma membrane (PM). Short and long-range arrays of the matrix protein were present and consisted of subunits in which the spacing was $\sim 8 \mathrm{~nm}$. In regions where the matrix protein was present along the interior of the plasma membrane, well-ordered arrays of the fusion $(\mathrm{F})$ and hemagglutinin $(\mathrm{H})$ glycoproteins were present on the surface. In addition, the ribonucleoprotein complex (RNP) appeared to associate directly with the ordered arrays of the matrix protein, indicating a coordinated recruitment to sites of virus assembly. Direct visualization of $\mathrm{MeV}$-infected cells by cryo-ET has proved valuable for determining the native organization of the M protein, glycoproteins, and ribonucleoprotein (RNP) at both sites of virus assembly and in released virus particles. 


\section{References:}

[1] MA Brindley, et al. Journal of Virology 87(21) (2014), p. 11693-11703.

[2] L Liljeroos, et al. Proceedings of the National Academy of Sciences of the United States of America 108(44) (2011) p. 18085-18090.

[3] T Paal, et al. Journal of Virology 83(20) (2009), p. 10480-10493.

[4] JD Strauss, et al. Journal of Virology 90(3) (2015) p. 1507-1521.

[5] CM Hampton, et al. Nature Protocols 12(1) (2017), p. 150-167.

[6] DN Mastronarde. Journal of Structural Biology 152(1) (2005) p. 36-51.

[7] JR Kremer, DN Mastronarde, and JR McIntosh, Journal of Structural Biology 116(1)(1996), p. 7176.

[8] We would like to thank the Robert P. Apkarian Integrated Electron Microscopy Core of Emory University for microscopy services and support. This work was supported in part by Emory University, Children's Healthcare of Atlanta, and the Georgia Research Alliance to E.R.W.; the Center for AIDS Research at Emory University (P30 AI050409); the James B. Pendleton Charitable Trust to E.R.W.; public health service grants R01AI083402 to R.K.P, F32GM112517 to J.D.S, and NSF grant 0923395 to E.R.W. 